\title{
A!
}

This is an electronic reprint of the original article.

This reprint may differ from the original in pagination and typographic detail.

Sihvola, Ari; Tzarouchis, Dimitrios C.; Ylä-Oijala, Pasi; Wallén, Henrik; Kong, Beibei

\section{Resonances in small scatterers with impedance boundary}

Published in:

Physical Review B

DOI:

10.1103/PhysRevB.98.235417

Published: 17/12/2018

Document Version

Publisher's PDF, also known as Version of record

Please cite the original version:

Sinvola, A., Tzarouchis, D. C., Ylä-Oijala, P., Wallén, H., \& Kong, B. (2018). Resonances in small scatterers with impedance boundary. Physical Review B, 98(23), [235417]. https://doi.org/10.1103/PhysRevB.98.235417

This material is protected by copyright and other intellectual property rights, and duplication or sale of all or part of any of the repository collections is not permitted, except that material may be duplicated by you for your research use or educational purposes in electronic or print form. You must obtain permission for any other use. Electronic or print copies may not be offered, whether for sale or otherwise to anyone who is not an authorised user. 


\title{
Resonances in small scatterers with impedance boundary
}

\author{
Ari Sihvola, Dimitrios C. Tzarouchis, Pasi Ylä-Oijala, Henrik Wallén, and Beibei Kong \\ Department of Electronics and Nanoengineering, Aalto University School of Electrical Engineering, 00076 Espoo, Finland
}

(Received 24 May 2018; revised manuscript received 22 November 2018; published 17 December 2018)

\begin{abstract}
With analytical (generalized Mie scattering) and numerical (integral-equation-based) considerations we show the existence of strong resonances in the scattering response of small spheres with a lossless impedance boundary. With increasing size, these multipolar resonances are damped and shifted with respect to the magnitude of the surface impedance. The electric-type resonances are inductive and the magnetic ones capacitive. Interestingly, these subwavelength resonances resemble plasmonic resonances in small negative-permittivity scatterers but occur in two families unlike the only-electric multipoles in the plasmonic case. The fundamental dipolar mode is also analyzed from the point of view of surface currents and the effect of the change of the shape into a nonspherical geometry. For dissipative impedance scatterers, maximum absorption is shown to result in the matched-impedance case.
\end{abstract}

DOI: 10.1103/PhysRevB.98.235417

\section{INTRODUCTION}

Slight perturbations in a system usually lead to small changes in its response function. In electromagnetic scattering, a good example is Rayleigh scattering, which means that the total scattered power of a particle that is small compared with the wavelength is proportional to the sixth power of its linear size, and thus vanishes predictably for tiny objects. But there are exceptions: A plasmonic subwavelength particle may have a very strong scattering response. This happens, for example, when the relative permittivity of a sphere hits the value -2 , and the particle is capable of supporting a localized dipolarlike surface plasmon. The magnitude of the resonance is in practice attenuated by material losses and radiation damping but can in principle reach very large values for spheres with a diameter that is much smaller than the wavelength of the incident radiation. In this paper, we report on a similar phenomenon that can be found in other types of small scatterers: Our analysis shows that particles with a special type of impedance boundary can have scattering and extinction efficiencies that grow without limit when their size decreases in the subwavelength domain. This phenomenon may have fundamental implications regarding the scattering by optically small objects.

In electromagnetics, a multitude of boundary conditions exists that can be classified into different categories [1]. Among those, much used are the perfect electric conductor (PEC) and perfect magnetic conductor (PMC) boundaries, on which the tangential electric (PEC) or tangential magnetic (PMC) field has to vanish. These conditions are special cases of the impedance boundary condition (IBC) which requires the following relation between the tangential electric $\left(\mathbf{E}_{t}\right)$ and magnetic $\left(\mathbf{H}_{t}\right)$ fields,

$$
\mathbf{E}_{t}=Z_{s} \mathbf{n} \times\left(\eta_{0} \mathbf{H}_{t}\right)
$$

on the surface with unit normal vector $\mathbf{n}$. The surface impedance is a naked number, having units of free-space impedance $\eta_{0}=\sqrt{\mu_{0} / \varepsilon_{0}}$. For the impedance surface to be lossless, $Z_{s}$ has to be purely imaginary (see Sec. 3.6 in Ref. [2]). A passive (dissipative) surface has a positive real part of $Z_{s}$, and correspondingly the negative real part means an active surface.

The history of the IBC concept reaches back to the 1940's $[3,4]$ when it was introduced in connection with the analysis of radio-wave propagation over ground. The scattering problem involving IBC objects has been treated in some studies in the past (see Sec. 10.4 in Ref. [5], and Refs. [6,7]), but it seems that the fundamental phenomenon of resonance modes in small particles had not received attention earlier. The understanding of such mechanisms in the scattering problem opens up possibilities to tailor structures with desired electromagnetic responses. This complements other approaches that exist to engineer the scattering characteristics of material objects, such as metasurface-based manipulations [8-10] and various principles to reduce visibility, such as mantle cloaking [11].

\section{SCATTERING BY AN IBC SPHERE}

Let us first compute the interaction of an IBC sphere with an incident electromagnetic plane wave in free space. The incident field will be scattered from the sphere, and the scattered field can be expanded in an infinite series of spherical harmonic functions. The expansion coefficients follow from the boundary condition at the impenetrable surface of the sphere. Following mutatis mutandis the classical Lorenz-Mie analysis, we arrive at the electric $\left(a_{n}\right)$ and magnetic $\left(b_{n}\right)$ scattering coefficients,

$$
\begin{gathered}
a_{n}=\frac{x \mathrm{j}_{n-1}(x)-n \mathrm{j}_{n}(x)+i Z_{s} x \mathrm{j}_{n}(x)}{x \mathrm{~h}_{n-1}^{(1)}(x)-n \mathrm{~h}_{n}^{(1)}(x)+i Z_{s} x \mathrm{~h}_{n}^{(1)}(x)}, \\
b_{n}=\frac{x \mathrm{j}_{n-1}(x)-n \mathrm{j}_{n}(x)+\left(i / Z_{s}\right) x \mathrm{j}_{n}(x)}{x \mathrm{~h}_{n-1}^{(1)}(x)-n \mathrm{~h}_{n}^{(1)}(x)+\left(i / Z_{s}\right) x \mathrm{~h}_{n}^{(1)}(x)} .
\end{gathered}
$$

Here, $x=2 \pi a / \lambda$ is the size parameter of the sphere with radius $a$, and $\mathrm{j}_{n}$ and $\mathrm{h}_{n}^{(1)}$ are the spherical Bessel and 

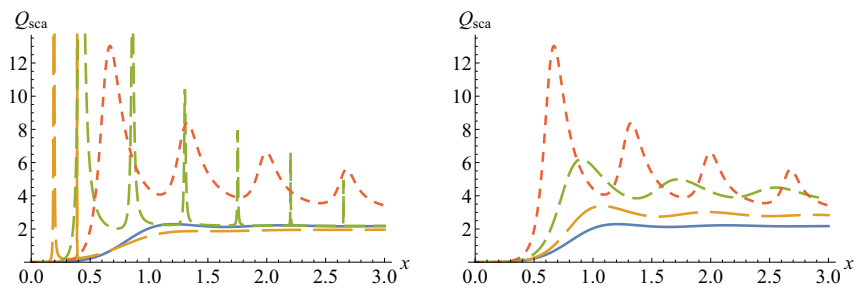

FIG. 1. Scattering (extinction) efficiency of lossless impedance spheres as functions of size parameter $x$, for certain values of $X_{s}$, which is the (negative of the) normalized surface reactance. Left: Solid blue, $X_{s}=0$ (PEC); long-dashed orange, $X_{s}=-0.2$; shortdashed green, $X_{s}=-0.5$; dotted red, $X_{s}=-1$. Right: Solid blue, $X_{s}= \pm \infty$ (PMC); long-dashed orange, $X_{s}=-5$; short-dashed green, $X_{s}=-2$; dotted red, $X_{s}=-1$.

Hankel (of the first kind) functions of order $n$. The convention $\exp (-i \omega t)$ is applied to map the sinusoidal time dependence into complex numbers. From these coefficients, the scattering (sca), extinction (ext), and absorption (abs) efficiencies can be computed according to the same principle as with classical efficiencies of penetrable spheres (see Sec. 4.4 in Ref. [12]),

$$
\begin{gathered}
Q_{\text {sca }}=\frac{2}{x^{2}} \sum_{n=1}^{\infty}(2 n+1)\left(\left|a_{n}\right|^{2}+\left|b_{n}\right|^{2}\right), \\
Q_{\text {ext }}=\frac{2}{x^{2}} \sum_{n=1}^{\infty}(2 n+1) \operatorname{Re}\left\{a_{n}+b_{n}\right\} \\
Q_{\text {abs }}=Q_{\text {ext }}-Q_{\text {sca }} .
\end{gathered}
$$

The efficiency is a dimensionless figure of merit, e.g., the scattering efficiency is the scattering cross section divided by the geometrical cross section of the particle. The series (4) and (5) converge. The larger the sphere in terms of wavelength, the more terms are needed. We use the Wiscombe criterion for the necessary number of terms $\left(N_{\max }=x+4 \sqrt[3]{x}+2\right)$ to truncate the series [13].

With this mathematical equipment, we can calculate the scattering and extinction behavior of spheres with arbitrary surface impedance and size. For lossy scatterers $\left(Z_{s}\right.$ has a nonzero real part), all three efficiencies are different while in the lossless case ( $Z_{s}$ is purely imaginary), the absorption efficiency vanishes and $Q_{\text {sca }}=Q_{\text {ext }}$. Following the notation of circuit theory, we write the surface impedance as

$$
Z_{s}=R_{s}-i X_{s}
$$

into the surface resistance $R_{s}$ and surface reactance $X_{s}$. The reactance $X_{s}$ is positive for inductive surfaces, and negative for capacitive. The particularly interesting finding from our studies concerns lossless scatterers for which the surface impedance is $Z_{s}=-i X_{s}$. We plot the scattering efficiencies of IBC spheres as functions of size parameter $x$ for different values of the surface impedance in Fig. 1. Due to the lossless character of the sphere, the scattering efficiency equals the extinction efficiency.

As to their scattering efficiency, the PEC $\left(Z_{s}=0\right)$ and PMC $\left(1 / Z_{s}=0\right)$ spheres behave identically (the two blue curves in Fig. 1). However, the functional form of the responses is far from trivial for intermediate surface impedance
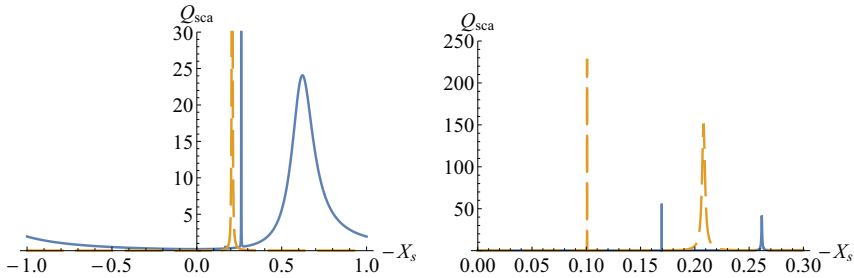

FIG. 2. Scattering (extinction) efficiency of lossless impedance spheres as functions of the surface reactance parameter $X_{s}$. The size parameter is $x=0.5$ (solid blue) and $x=0.2$ (long-dashed orange). Due to the broad range of $X_{s}$ in the left-hand side figure, very highorder resonances cannot be distinguished.

values. As the value of the reactance $X_{s}$ decreases from the PMC limit, a gradual increase in scattering amplitude takes place over the entire range. The evolution leads to oscillations, and once the surface reactance reaches small (negative) values, the whole curve is dominated by the resonances. In the PEC limit ( $X_{s}$ is close to zero), the resonances, riding on top of the gently rolling PEC curve, become vanishingly narrow.

The broadest (dipolar) resonance for very small spheres appears at $-X_{s} \approx x$, and the higher-order modes follow with $-X_{s} \approx x / n$ for integers $n$. Figure 2 shows a closer view of the resonances, showing the positions of quadrupolar and octopolar modes for size parameters $x=0.5$ and $x=0.2$. The amplitude of the resonances is $\max \left\{Q_{\text {sca }}\right\} \approx 2(2 n+1) x^{-2}$, being 24,40 , and 64 for the three lowest modes in the case of $x=0.5$, and 150, 250, and 350 for $x=0.2$.

Figure 3 illustrates the scattering characteristics as a function of the size parameter and the surface impedance. The effect of increasing sphere size is to soften the resonances and shift their position towards larger values of the imaginary part of the surface impedance. Two clusters of resonance

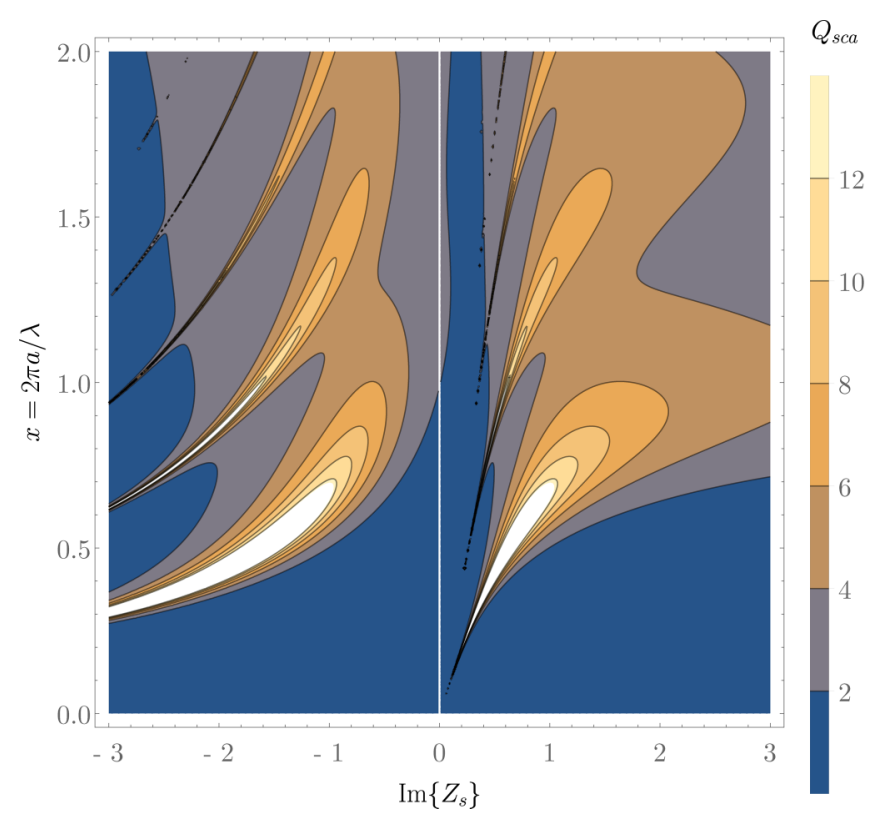

FIG. 3. A contour plot of the scattering efficiency of an impedance sphere as function of its surface reactance and the size parameter $x$. 

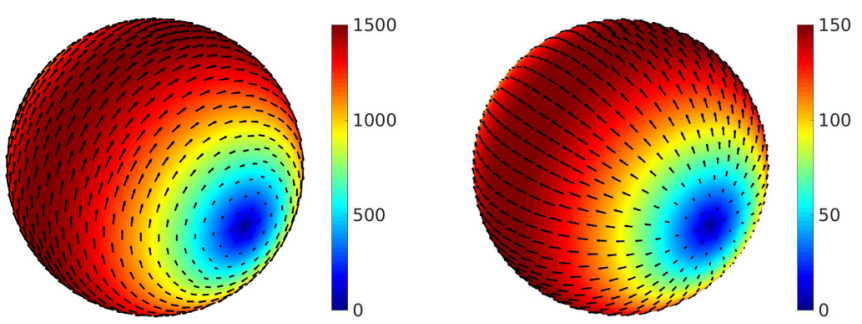

FIG. 4. The imaginary part of the electric surface current (left) and the real part of the magnetic surface current (right) for an IBC sphere with size parameter $x=0.1$ and homogeneous scalar surface impedance $Z_{s}=i 0.101$. The incident wave is propagating upwards from the bottom.

modes exist, one for positive and one for negative $X_{s}$. The resonances at $\operatorname{Im}\left\{Z_{s}\right\}>0$ are due to the maxima of the $b_{n}$ Mie coefficients (3), and hence are magnetic-type resonances, while the resonances for negative $\operatorname{Im}\left\{Z_{s}\right\}$ arise from $a_{n}(2)$, being of electric type. Despite the different visual appearances of the two clusters, they follow the symmetry

$$
Q_{\text {sca }}\left(x, X_{s}\right)=Q_{\text {sca }}\left(x,-1 / X_{s}\right) .
$$

To gain an understanding of the mode pattern of the lowest resonance, Fig. 4 displays the induced electric $\mathbf{J}_{s}$ and magnetic $\mathbf{M}_{s}$ surface currents on the sphere for the case $x=0.1$ and $Z_{s}=i 0.101$, with a plane-wave excitation. The surface currents are connected to the tangential fields as $\mathbf{J}_{s}=\mathbf{n} \times \mathbf{H}$ and $\mathbf{M}_{s}=-\mathbf{n} \times \mathbf{E}$. The current distributions show clearly a magnetic dipole type of structure (circulating electric current). Due to the boundary condition (1) where the surface impedance is imaginary, the currents have to be in $90^{\circ}$ phase shifted, and also rotated by $90^{\circ}$ on the sphere surface. The tenfold magnitude of the electric current compared with the magnetic follows from the amplitude of the surface reactance. [The figures display only the imaginary (real) part of $\mathbf{J}_{s}\left(\mathbf{M}_{s}\right)$; the out-of-phase components are around 3000 times smaller.]

The resonance structure of small IBC spheres, although resembling that of subwavelength plasmonic scatterers, has its own character. While plasmonic resonances are due only to the electric multipoles, here the electric and magnetic resonances are dual as seen in Eq. (8).

\section{DISSIPATIVE SCATTERERS}

When the surface impedance $Z_{s}$ has a real part, the surface is no longer lossless. Hence also the three efficiencies in (4)-(6) are different. For passive surfaces (the real part of $Z_{s}$ is positive), there is absorption $\left(Q_{\text {abs }}>0\right)$, and in the case of active surfaces, absorption is negative. The interplay between scattering, absorption, and extinction for lossy impedance spheres is depicted in Fig. 5 for two cases: $Z_{s}$ has real and positive values 1 and 10 .

The dominance of absorption over scattering is conspicuous for small spheres in Fig. 5: The extinction is mainly due to absorption when $x$ is small. This phenomenon is also seen in the Taylor expansions of the scattering efficiencies (4) and (5). The ordinary Rayleigh scattering dependence $Q_{\text {sca }} \approx(16 / 3) x^{4}$ holds for scattering, while the absorption
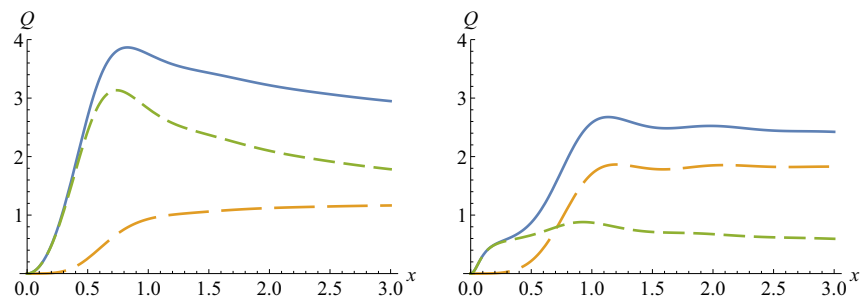

FIG. 5. Extinction (solid blue), scattering (long-dashed orange), and absorption (short-dashed green) efficiencies for spheres with lossy surface impedance as a function of the size parameter: $Z_{s}=1$ (left), $Z_{s}=10$ (right).

efficiency has a square dependence on the size parameter,

$$
Q_{\mathrm{abs}} \approx 6\left(Z_{s}+1 / Z_{s}\right) x^{2} .
$$

This square dependence of absorption efficiency on the size parameter of IBC spheres differs from the corresponding behavior of the absorption of lossy penetrable dielectric spheres in which the dependence is linear in the small-particle limit [14], and the maximum absorption occurs when the losses match the radiative damping term $[15,16]$. For small spheres with fixed $x$, the maximum absorption takes place for increasing $Z_{s}$ or $1 / Z_{s}$, but as the size parameter is larger than 0.4 , the maximum is strongest for the "matched-impedance" case $Z_{s}=1$ (for which the scattering achieves its minimum). Such a sphere is an example of a zero-backscattering object [17].

The efficiencies are invariant with respect to the change $Z_{s} \rightarrow 1 / Z_{s}$. Therefore (8) can be written for general complex IBC spheres as $Q\left(x, Z_{s}\right)=Q\left(x, 1 / Z_{s}\right)$, valid for all three efficiency quantities and $Z_{s}=R_{s}-i X_{s}$.

Figure 6 shows contour plots of the three efficiencies as functions of the size parameter and the (real-valued) surface impedance. In agreement with the extinction paradox (see Sec. 4.4.3 in Ref. [12], and Ref. [18]), the extinction efficiency approaches the value 2 for large spheres, independent of the surface impedance. The convergence can be slow if $\left|Z_{s}\right| \approx 1$ : To be within $1 \%$ of this limiting value, $x$ needs to be around 1000 .

\section{NONSPHERICAL OBJECTS}

The sphere is an extravagantly symmetric shape. It is fair to raise the question whether the IBC resonances remain if the spherical symmetry is broken. As an answer, we compute the scattering behavior in the vicinity of the resonance of the magnetic dipole for an IBC superspherical object, using the numerical surface-integral-equation method based on the electric field integral equation formulation for IBC scatterers $[19,20]$. (Appendix A gives the computational details.) The surface of such an object is defined by

$$
|x|^{p}+|y|^{p}+|z|^{p}=a^{p} .
$$

The value $p=2$ reproduces a sphere with radius $a, p=1$ an octahedron, and for increasing $p$, the shape approaches a cube [21]. Figure 7 shows how the scattering response, in particular, the position of the main resonance, shifts with the shape of the object. Not surprisingly, the spherical geometry gives an extremum. As an additional numerical illustration, 

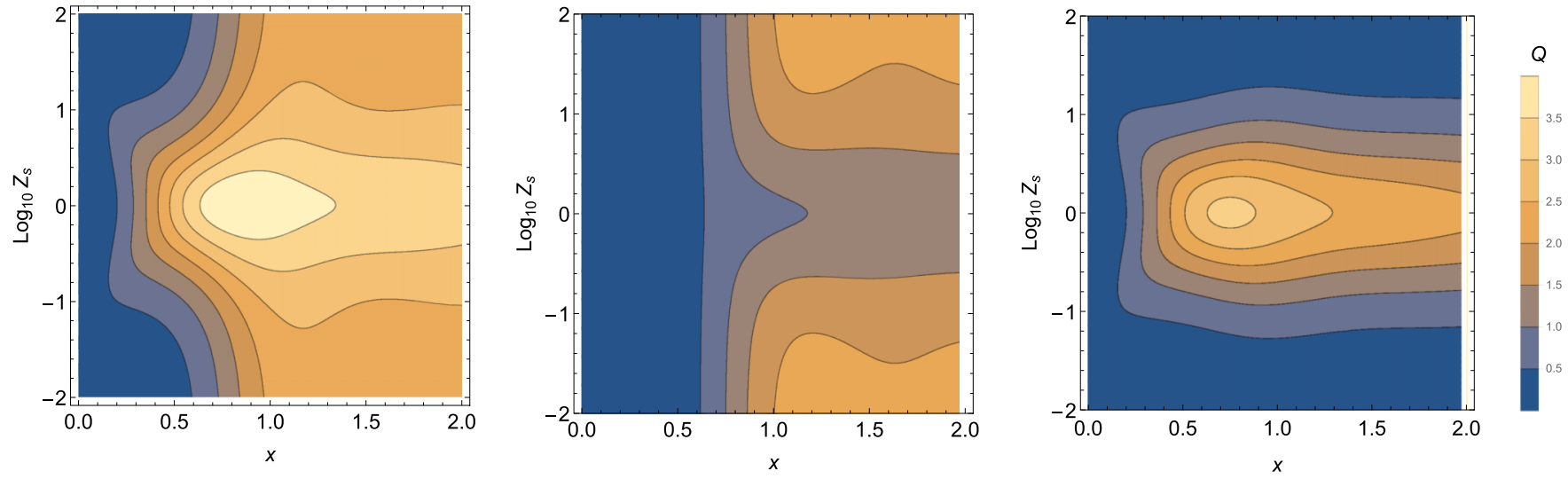

FIG. 6. Extinction (left), scattering (center), and absorption (right) efficiencies of a lossy IBC sphere as functions of the size parameter (horizontal axis $x$ ) and the (logarithmic) surface impedance.

Appendix B shows the computed resonance structure for an ellipsoidal IBC object.

\section{DISCUSSION}

The question about the material realization of these scatterers remains. In engineering electromagnetics, the boundary condition has been used as an approximation to interfaces between materially strongly contrasting media, often with success, such as in the response of metal surfaces in the microwave region. However, interfaces over which the material parameters only change moderately cannot be accurately described by a boundary condition due to the fact that the relation between the tangential electric and magnetic fields depends on the incidence angle and polarization of the incident wave. A remedy to the synthesis problem is the so-called

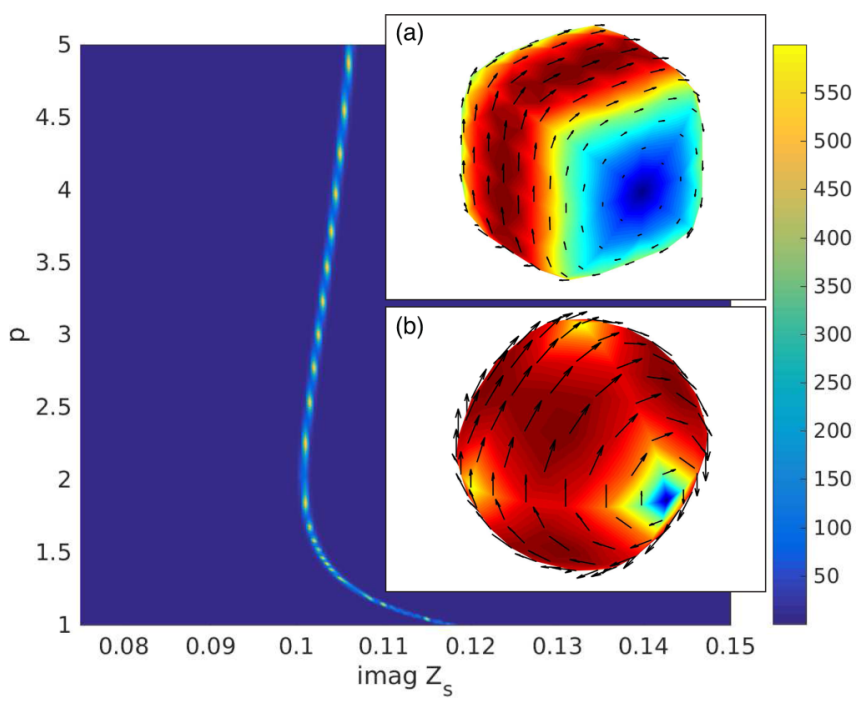

FIG. 7. The magnitude of the scattering efficiency of a small lossless IBC quasispherical particle in the plane of the surface impedance and the supersphere parameter $p$. Throughout the $p$ scale, the volume of the particle is the same as the volume of a sphere with $p=2$ for which $x=0.1$. The insets show the electric surface current distributions for cases (a) $p=5$ and (b) $p=1.2$. waveguiding material [22], which is an anisotropic medium that has large components in the permittivity and permeability dyadics in one direction. Cut perpendicular to this direction, the material surface behaves as an impedance surface since the large values of the normally directed material parameter components force the longitudinal fields to vanish, and the fields in the material remain transversal. Adding a parallel metallic plate at a certain depth, the waves are reflected and travel as in a waveguide, and the field relation can be manipulated by varying the transversal permittivity and permeability components of the medium. Another approach to materialize a structure mimicking the surface reactance is to make use of frequency-selective-surface (FSS) principles [23,24] and carve a regular subwavelength pattern of holes on a conducting metallic surface, thus manipulating the ratio between the averaged electric and magnetic fields to produce the desired surface impedance. For instance, the idea of nanoparticles coated with a thin layer of graphene [25] can be embraced from the point of view of the IBC approach with its two classes of resonances, thus exploiting both the inductive and capacitive regions of graphene spectrum.

As a final note, the fascinating resonance spectrum described in this paper is given as a function of the size parameter which is essentially a product of frequency and absolute size. Hence it can describe scattering functions of the sphere for varying frequency or a single-frequency response of a distribution of spheres of varying size.

\section{APPENDIX A: COMPUTATIONAL DETAILS IN THE NUMERICAL EVALUATIONS OF SCATTERING}

The scattering computations in this paper are based on the surface-integral-equation approach. It can be applied for arbitrary-shaped impedance boundary objects. In this approach, first the surface equivalence principle is used to express the scattered electromagnetic fields in terms of the equivalent electric and magnetic surface current densities. Then IBC is applied to express the magnetic current in terms of the electric one. This allows us to eliminate the magnetic current from the equations. The resulting integral equations are discretized and converted to a matrix equation using Galerkin's testing procedure and Rao-Wilton-Glisson (RWG) 


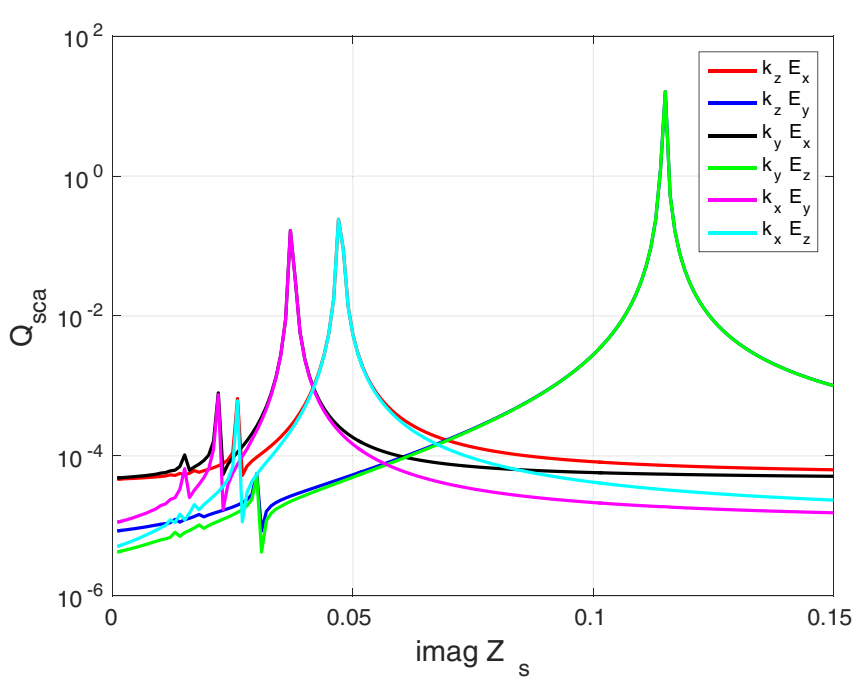

FIG. 8. Scattering (extinction) efficiency of a small lossless impedance ellipsoid as functions of the (imaginary part of the) surface impedance, for six different excitations.

functions $[20,26]$. To simplify the numerical implementations, IBC is enforced in the weak sense [19]. For the ellipsoid problem (Fig. 8), the discretization has been done with 720 elements (triangles), and the number of unknowns (edges in the triangle mesh) was 1080.

\section{APPENDIX B: RESONANCE STRUCTURE IN NONSYMMETRIC OBJECTS}

In order to see how the scattering spectrum of an impedance boundary object is affected by morphological changes, we compute the scattering efficiency $Q_{\text {sca }}$ for an ellipsoid with three different semiaxis lengths. This breaks the symmetries of the sphere and the supersphere that were analyzed in the main text.

Figure 8 shows the scattering efficiency spectra for an electrically small ellipsoid with a lossless impedance bound- ary condition $\left(Z_{s}\right.$ is purely imaginary). The semiaxes of the ellipsoid are

$$
k a_{x}=0.1, \quad k a_{y}=0.05, \quad k a_{z}=0.033,
$$

with $k=2 \pi / \lambda$.

The magnetic dipole resonance, which for a spherical scatterer occurred at $Z_{s}=0.1 i$ for a sphere with a size parameter $x=0.1$ as shown in the main text, is now split into three main resonances. These happen for values around $\operatorname{Im}\left\{Z_{s}\right\}=0.115$, 0.047 , and 0.036 , depending on the excitation.

Due to the fact that there are six basic different excitation constellations (the wave can be incident for each of the three axis directions, and for each of these, the electric field can take any of the two perpendicular polarizations), the figure shows six different curves. However, these six occur in three closely similar pairs, which is due to the small electrical size of the scatterer.

For example, the curves displaying the strongest resonance are for a $z$-propagating wave with a $y$-polarized electric field (dark blue curve) which falls very closely on the curve corresponding to the $y$-propagating wave with the electric field polarized along the $z$ axis (green curve). In both of these cases, the magnetic field is $x$ polarized into which direction the ellipsoid has its largest semiaxis, corroborating the conclusion that the resonance is due to a magnetic multipole. The wave propagation direction obviously does not have a strong effect on the resonance curve, due to the smallness of the particle over which there is not much phase delay for the wave.

\section{APPENDIX C: IMPEDANCE BOUNDARY IN GRAPHENE-COATED NANOSPHERES}

As a possible realization of the IBC particles, we suggest graphene-coated nanoparticles. Graphene offers capacitive and inductive responses at terahertz frequencies. The characteristic bulk conductivity of graphene is usually modeled as a combination of intra- and interband transitions,

$$
\sigma(\omega)=\sigma_{\text {intra }}(\omega)+\sigma_{\text {inter }}(\omega)
$$

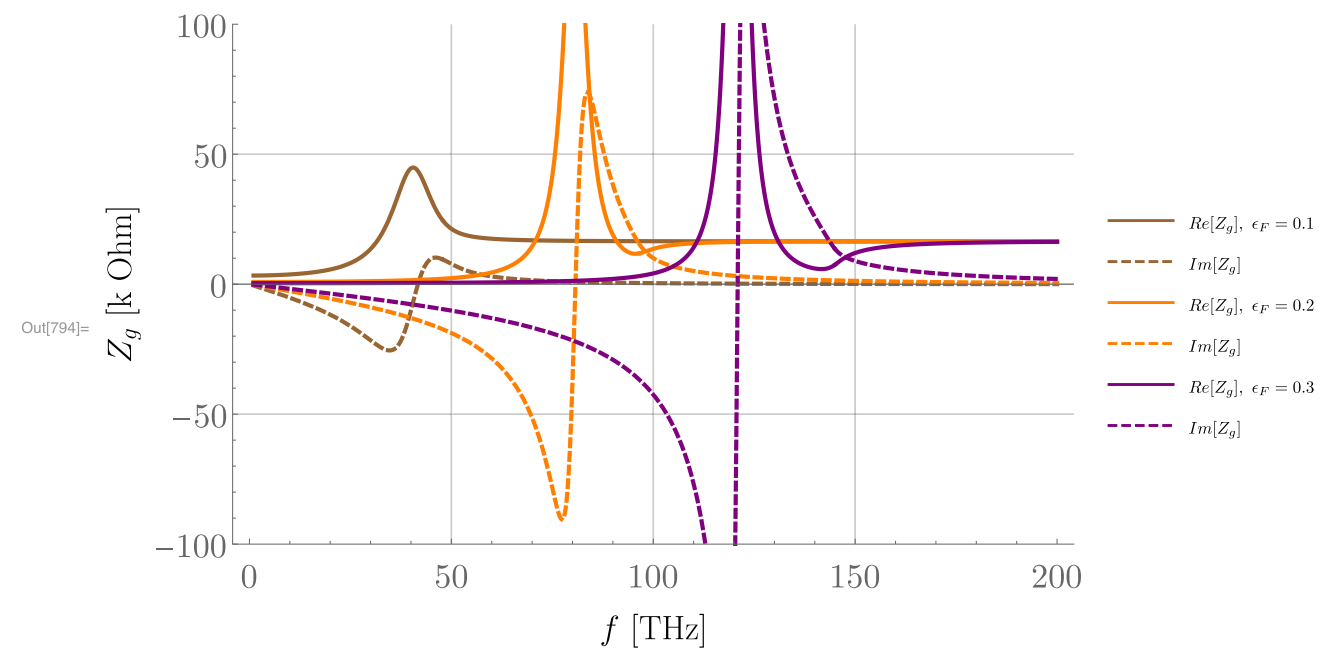

FIG. 9. The real (solid lines) and imaginary (dashed lines) of the surface impedance of a graphene sheet in $\mathrm{k} \Omega$. Varying the chemical potential shifts and modifies the spectrum: $\epsilon_{\mathrm{F}}=0.1 \mathrm{eV}$ (brown), $\epsilon_{\mathrm{F}}=0.1 \mathrm{eV}$ (orange), and $\epsilon_{\mathrm{F}}=0.1 \mathrm{eV}$ (purple). Graphene mobility is assumed to be $\mu=0.5 \mathrm{~m}^{2} /(\mathrm{V} \mathrm{s})$. 
With the assumption that the chemical potential of graphene $\epsilon_{\mathrm{F}}$ is much larger than the scale factor $k_{\mathrm{B}} T$, we can approximate the graphene conductivity as [27]

$$
\sigma_{\text {intra }} \approx \frac{i e^{2} k_{\mathrm{B}} T}{\pi \hbar^{2}(\omega+i 2 \Gamma)}\left[\frac{\epsilon_{\mathrm{F}}}{k_{\mathrm{B}} T}+2 \log \left(e^{-\frac{\epsilon_{\mathrm{F}}}{k_{\mathrm{B}} T}}+1\right)\right]
$$

and

$$
\sigma_{\text {inter }} \approx \frac{i e^{2}}{4 \pi \hbar} \log \left(\frac{2\left|\epsilon_{\mathrm{F}}\right|-(\omega+i 2 \Gamma) \hbar}{2\left|\epsilon_{\mathrm{F}}\right|+(\omega+i 2 \Gamma) \hbar}\right) .
$$

Here, the parameters are as follows: $\Gamma=\frac{e u_{\mathrm{F}}^{2}}{\mu \epsilon_{\mathrm{F}}}$ is the phenomenological scattering rate (inverse of relaxation time), $\mu=0.5 \mathrm{~m}^{2} /(\mathrm{Vs})$ is value used in our computations for the graphene mobility, $u_{\mathrm{F}}=10^{6} \mathrm{~m} / \mathrm{s}$ the Fermi velocity, $k_{\mathrm{B}}=1.3806 \times 10^{-23} \mathrm{~J} / \mathrm{K}$ is the Boltzmann's constant, $T=$ $300 \mathrm{~K}$ is the absolute temperature, and $e=1.602 \times 10^{-19} \mathrm{~A} \mathrm{~s}$ is the electron charge.
With this model, we plot Fig. 9 which illustrates the surface impedance of a graphene sheet for different chemical potential values $\epsilon_{\mathrm{F}}$. We can observe two regions, one with inductive and one with capacitive characteristics, situated below and above a central resonant region, respectively. The inductive region ("plasmonic") generally experiences smaller ohmic losses (real part of impedance) allowing the direct exploitation of this region for localized plasmonic resonances as shown in Ref. [25]. However, also capacitive resonances can occur, just above the region of strong resonance. Admittedly, this capacitive region experiences much larger losses than in the band below the resonance. However, especially for large $\epsilon_{\mathrm{F}}$ values, there is a region where the losses achieve a minimum. This window might allow the implementation of a new class of "dielectric" resonances in plasmons, thus generalizing the present-day focus on only plasmonic resonances exploited in connection with graphene sheets $[25,28]$.
[1] I. V. Lindell and A. Sihvola, IEEE Trans. Antennas Propag. 65, 4656 (2017).

[2] I. V. Lindell, Methods for Electromagnetic Field Analysis (Oxford University Press, Oxford, UK, 1992).

[3] A. N. Shchukin, Propagation of Radio Waves (Svyazizdat, Moscow, 1940).

[4] M. A. Leontovich, Bull. Acad. Sci. USSR, Phys. Ser. 8, 16 (1944) (in Russian).

[5] J. D. Jackson, Classical Electrodynamics (Wiley, New York, 1999).

[6] A. W. Glisson, Radio Sci. 27, 935 (1992).

[7] G. Ya. Slepyan, S. A. Maksimenko, A. Lakhtakia, O. Yevtushenko, and A. V. Gusakov, Phys. Rev. B 60, 17136 (1999).

[8] M. Selvanayagam and G. V. Eleftheriades, Phys. Rev. X 3, 041011 (2013).

[9] S. Vellucci, A. Monti, A. Toscano, and F. Bilotti, Phys. Rev. Appl. 7, 034032 (2017).

[10] S. B. Glybovski, S. A. Tretyakov, P. A. Belov, Y. S. Kivshar, and C. R. Simovski, Phys. Rep. 634, 1 (2016).

[11] A. Alù, Phys. Rev. B 80, 245115 (2009).

[12] C. F. Bohren and D. R. Huffman, Absorption and Scattering of Light by Small Particles (Wiley, New York, 1983).

[13] W. J. Wiscombe, Appl. Opt. 19, 1505 (1980).

[14] H. Wallén, H. Kettunen, and A. Sihvola, Radio Sci. 50, 18 (2015).
[15] M. I. Tribelsky and B. S. Luk'yanchuk, Phys. Rev. Lett. 97, 263902 (2006).

[16] D. C. Tzarouchis, P. Ylä-Oijala, and A. Sihvola, Phys. Rev. B 94, 140301(R) (2016).

[17] I. V. Lindell, A. H. Sihvola, P. Ylä-Oijala, and H. Wallén, IEEE Trans. Antennas Propag. 57, 2725 (2009).

[18] L. Brillouin, J. Appl. Phys. 20, 1110 (1949).

[19] Ismatullah and T. F. Eibert, IEEE Trans. Antennas Propag. 57, 2084 (2009).

[20] P. Ylä-Oijala, J. Lappalainen, and S. Järvenpää, IEEE Trans. Antennas Propag. 66, 487 (2018).

[21] D. C. Tzarouchis, P. Ylä-Oijala, T. Ala-Nissila, and A. Sihvola, J. Opt. Soc. Am. B 33, 2626 (2016).

[22] I. V. Lindell and A. H. Sihvola, IEEE Trans. Antennas Propag. 54, 3669 (2006).

[23] B. A. Munk, Frequency Selective Surfaces: Theory and Design (Wiley, New York, 2000).

[24] F. Liu, S. Xiao, A. Sihvola, and J. Li, IEEE Trans. Antennas Propag. 62, 6274 (2014).

[25] T. Christensen, A.-P. Jauho, M. Wubs, and N. A. Mortensen, Phys. Rev. B 91, 125414 (2015).

[26] S. Rao, D. Wilton, and A. Glisson, IEEE Trans. Antennas Propag. 30, 409 (1982).

[27] G. W. Hanson, J. Appl. Phys. 103, 064302 (2008).

[28] X. Xiao, X. Li, J. D. Caldwell, S. A. Maier, and V. Giannini, Appl. Mater. Today 12, 283 (2018). 